\title{
Características morfológico-funcionales, diversidad arbórea, tasa de crecimiento y de secuestro de carbono en especies y ecosistemas de Polylepis del sur de Ecuador
}

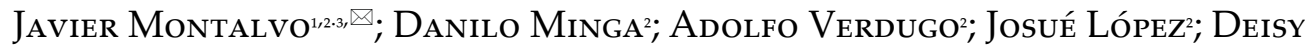 \\ Guazhambo $^{23}$; Diego Pacheco'; David Siddons²; Antonio Crespo² \& Edwin Zárate ${ }^{2}$ \\ ${ }^{1}$ Departamento de Ecología y Biología Animal, Universidad de Vigo, España. ${ }^{2}$ Escuela de Biología, Ecología y Gestión, \\ Universidad del Azuay, Ecuador. ${ }^{3}$ Fundación Matrix, Investigación y Desarrollo Sostenible, España.
}

\begin{abstract}
Resumen. Las especies del género Polylepis son los árboles más importantes de los bosques Andinos de gran altitud, distribuidos en un área con gran variación climática desde Venezuela hasta la Argentina. En el clima húmedo del Macizo del Cajas (sur de Ecuador), cuatro especies nativas de Polylepis forman bosques poco conocidos entre 2800 y $4400 \mathrm{~m}$ s. n. m.: P. reticulata, $P$. incana, $P$. weberbaueri y $P$. lanuginosa (esta última, endémica de Ecuador). Se estudiaron características morfo-funcionales de estas especies y de otra introducida (P. racemosa), y atributos a nivel de población y de ecosistema, relevantes para comprender su distribución local y los efectos del cambio global. El área foliar disminuye con la altitud, y P. lanuginosa presenta una de las mayores del género Polylepis $\left(17.3 \mathrm{~cm}^{2}\right)$. El alto contenido de agua $(>55 \%)$ y la baja densidad de la madera del fuste de tres especies sugieren una respuesta a la limitación de la producción primaria por el frío o por la aridez climática. Los bosques de $P$. reticulata y $P$. incana presentan una diversidad arbórea (índice de Shannon) moderada; los de $P$. lanuginosa, una relativamente alta, con máximos superiores a 3 bits. La diversidad arbórea disminuye de forma no lineal con el incremento de la densidad de Polylepis, siempre mayor a 400 individuos/ha y con una frecuencia superior a 1000 individuos/ha. La biomasa aérea es muy variable, y notable en algún caso (alrededor de $200 \mathrm{Mg} \mathrm{C} / \mathrm{ha}$ ). La tasa de crecimiento diametral de $P$. reticulata tras siete años es $1.2 \mathrm{~mm} /$ año y la tasa de secuestro de carbono promedio es $2.6 \pm 0.3 \mathrm{Mg} \mathrm{C} \cdot \mathrm{ha}^{-1}$. $\mathrm{año}^{-1}$, relevante para un género de lento crecimiento. La conservación de bosques de Polylepis es pertinente para preservar la biodiversidad altoandina amenazada y mitigar el cambio climático por su capacidad de retener carbono en biomasa.
\end{abstract}

[Palabras clave: área foliar específica, biomasa, bosque, contenido de agua de la madera, densidad de la madera, diversidad de especies, secuestro de carbono]

\begin{abstract}
Aвstract. Morphological-functional traits, tree diversity, growth rate and carbon sequestration in Polylepis species and ecosystems of southern Ecuador. The species of the genus Polylepis are the most important trees of high altitude woodlands in the Andes, an area with great climatic variation from Venezuela to Argentina. In the humid climate of the Cajas Massif (southern Ecuador), four native Polylepis species form little known woodlands between 2800 and $4400 \mathrm{~m}$ a.s.l.: P. reticulata, $P$. incana, $P$. weberbaueri and P. lanuginosa (the latter, endemic to Ecuador). Morphological-functional traits of these species and of another introduced (P. racemosa), and attributes at the population and ecosystem levels relevant to understanding their local distribution and the effects of global change were studied. Leaf area decreases with altitude, showing $P$. lanuginosa one of the largest of the genus Polylepis $\left(17.3 \mathrm{~cm}^{2}\right)$. High stem water content $(>55 \%)$ and low stem wood density of three species suggest an ecological response to primary production limitation due to cold and/or climatic aridity. Tree species diversity (Shannon index) is moderate in the woodlands of $P$. reticulata and $P$. incana and relatively high in $P$. lanuginosa ones, with maxima higher than 3 bits. Tree species diversity decreases non-linearly with increasing Polylepis tree density, always greater than 400 individuals/ha and frequently more than 1000 individuals/ha. Aboveground biomass is a very variable ecosystem attribute and remarkable in some woodlands (about 200 $\mathrm{Mg} \mathrm{C} / \mathrm{ha}$ ). The diameter growth rate at breast height of $P$. reticulata after seven years is $1.2 \mathrm{~mm} / \mathrm{year}$, and the average carbon sequestration rate is $2.6 \pm 0.3{\mathrm{Mg} \mathrm{C} . h a^{-1} \text {.year }}^{-1}$, outstanding for a slow-growing genus. Polylepis woodlands conservation is pertinent to preserve threatened high Andean biodiversity and mitigate climate change by its ability to retain carbon in biomass.
\end{abstract}

[Keywords: biomass, forest, species diversity, specific leaf area, stem water content, wood density, carbon sequestration]

Editor asociado: Daniel Renison 


\section{INTRODUCCIÓN}

Alrededor de 27 especies arbóreas integran el género Polylepis (Kessler and Schmidt-Lebuhn 2006; Mendoza and Cano 2012). Forman bosques en general dominados por una única especie de Polylepis situados a elevada altitud en la cordillera de los Andes, desde áreas tropicales de páramo de Venezuela hasta otras subtropicales del altiplano andino de Chile y la Argentina, y alcanzan su límite meridional en las sierras del centro argentino (Simpson 1979; Kessler and Schmidt-Lebuhn 2006; Renison et al. 2013). Casi $5000 \mathrm{~km}$ de distancia latitudinal conllevan una variabilidad amplia, tanto climática como de bosques con presencia de distintas especies de Polylepis. La mayoría del área original de Polylepis se perdió por actividades humanas en el último milenio y es uno de los bosques de montaña más amenazados del mundo (Kessler 2000; Fjeldså 2002). La deforestación en los Andes ecuatorianos es elevada, con una tasa anual de $-0.75 \%$; si se mantiene, existen proyecciones de desaparición total de la cobertura boscosa en los próximos 100 años (Tejedor Garavito et al. 2012, 2015).

Sin embargo, a pesar del alto interés de los bosques de Polylepis para la conservación (Tejedor Garavito et al. 2015), o de su distribución en páramos valiosos para la provisión de agua, a veces incluidos en parques del Sistema Nacional de Áreas Protegidas, no existen estudios ecológicos detallados de las características de todas las especies y bosques de Polylepis en Ecuador. Las poblaciones y bosques de Polylepis constituyen teselas o rodales aislados de pequeño tamaño que expresan una fragmentación intensa a escala de paisaje, y áreas de ocupación actual muy restringidas en algunos casos (Romoleroux 1992, 1996). Como en otras áreas andinas, el origen de la fragmentación y pérdida de bosque es antropogénico y consecuencia de fluctuaciones paleoclimáticas (Kessler 2000, 2002; Gosling et al. 2009; Rodríguez and Behling 2012; Purcell et al. 2014).

El conocimiento de las características de las especies y bosques de Polylepis en el sur de Ecuador es aun más limitado. En el Macizo del Cajas existen cuatro especies nativas de Polylepis (Minga et al. 2016). Forman bosques poco conocidos a lo largo de un gradiente altitudinal de $1500 \mathrm{~m}$, con presencia de rodales aislados en la matriz de páramo -dominada por pajonal - hasta unos 4300 m s. n. m. (Coblentz and Keating 2008). Las características morfo-funcionales de estas especies y los atributos de sus bosques a nivel de ecosistema (e.g., diversidad de especies, biomasa o servicio de secuestro de carbono) no han sido estudiados, aunque son relevantes para comprender su adaptación y su distribución local (e.g., Toivonen et al. 2014; Vásquez et al. 2014), y su conservación ante el cambio global (e.g., Cuykens et al. 2016). La conservación de bosques altoandinos es prioritaria por su mayor dificultad de restauración y regeneración natural (Cierjacks et al. 2007). Tanto el diseño y la implementación de proyectos de restauración ecológica de bosques de Polylepis como la valoración de sus servicios ecosistémicos se beneficiarían del conocimiento de atributos básicos de ecosistemas de referencia (Renison et al. 2011) y de una mejor comprensión de las características ecológicas y del valor adaptativo de las especies de Polylepis. Este conocimiento también contribuiría a contrarrestar los riesgos de degradación genética de las poblaciones de $P$. incana derivados de la proliferación de plantaciones de una especie exótica de crecimiento más rápido, $P$. racemosa, en el páramo andino de Ecuador (Segovia-Salcedo 2011).

Los objetivos principales de este trabajo realizado en el Macizo del Cajas son: 1) conocer algunas características morfofuncionales de las hojas y del fuste de cuatro especies nativas de Polylepis, de tres presentes en otros países $(P$. reticulata, $P$. weberbaueri y $P$. incana) y de una endémica de Ecuador $(P$. lanuginosa), y compararlas con las de una especie introducida ( $P$. racemosa); 2) describir la densidad de árboles de Polylepis, su biomasa aérea y la diversidad de especies arbóreas de bosques, y 3) estimar el crecimiento y la tasa de secuestro de carbono en un bosque de $P$. reticulata. Nuestra hipótesis de trabajo general es que las diferencias morfo-funcionales interespecíficas y de atributos ecosistémicos son relevantes para conocer los factores ambientales que controlan la distribución local de las especies de Polylepis, y la peculiar estructura y funcionamiento de sus bosques.

\section{Materiales y Métodos}

\section{Clima del área de estudio}

El Macizo del Cajas es un área que une dos cordilleras andinas en la provincia de Azuay, al sur de Ecuador. Estudiamos el núcleo del Macizo y Reserva de la Biosfera Cajas, ubicado 
aproximadamente entre las coordenadas $2^{\circ} 45^{\prime} \mathrm{S}-2^{\circ} 55^{\prime} \mathrm{S}$ y $79^{\circ} 08^{\prime} \mathrm{O}-7^{\circ} 24^{\prime} \mathrm{O}$, que incluye el Parque Nacional Cajas. Según una evaluación de los datos WorldClim (Hijmans et al. 2005), en el gradiente altitudinal donde prácticamente se concentra la distribución de los bosques de Polylepis (2800-4300 m s. n. m.), la temperatura media anual varía entre 4 y $12^{\circ} \mathrm{C}$. La temperatura mínima extrema anual $0^{\circ} \mathrm{C}$, se alcanza a $4200 \mathrm{~m} \mathrm{s.n}$. $\mathrm{m}$. y su promedio en este gradiente varía entre 7 y $-0.5^{\circ} \mathrm{C}$. Existen heladas, pero no son frecuentes ni intensas, y se limitan a breves períodos. La precipitación total anual supera los $800 \mathrm{~mm}$, con un pico de más de 1040 mm entre 3000 y 4000 m s. n. m., y al menos $900 \mathrm{~mm}$ hasta los $4300 \mathrm{~m} \mathrm{~s}$. n. m. Es probable que sea superior en el sector oriental del Macizo. Existen registros de 1200-1700 mm en algunas estaciones meteorológicas ubicadas a una altitud de alrededor de $3500 \mathrm{~m} \mathrm{s.} \mathrm{n.} \mathrm{m.}$ La lluvia se distribuye a lo largo de todos los meses sin un período marcadamente seco. Por tanto, el páramo del Macizo del Cajas presenta un clima tropical húmedo con temperatura fresca-fría, sin predominio de heladas y sin déficit en la disponibilidad de agua porque la precipitación y la evapotranspiración, limitada por el frío, mantienen un balance hídrico positivo (observación personal).

\section{Especies de Polylepis estudiadas}

Las características foliares son distintivas de las especies de Polylepis, más que las florales (Simpson 1979). Las especies estudiadas corresponden a los tres diferentes grupos morfológicos definidos por Simpson (1979) con base filogenética (Schmidt-Lebuhn et al. 2010). P. lanuginosa, es una especie endémica del centro y sur de Ecuador, diploide y con hojas mesomórficas. Está adscrita al grupo 'sericea', con numerosos pares de foliolos con pubescencia sericea o estrigosa en el envés. Se la considera una de las especies más primitivas del género (Mendoza and Cano 2012). P. reticulata y $P$. weberbaueri son diploides, con hojas xeromórficas; pertenecen al grupo 'reticulata', con pubescencia panosa. $P$. incana es diploide (Segovia-Salcedo and Quijia-Lamiña 2013), con hojas xeromórficas más delgadas que las especies del grupo 'sericea'. Pertenece al complejo 'incana', a menudo con solo un par de foliolos con pubescencia lanosa. P. racemosa también pertenece al grupo 'incana', pero sus hojas presentan una cutícula más fina que la de $P$. incana (Simpson 1979).

\section{Bosques de Polylepis estudiados}

La máxima distancia entre los bosques de las cuatro especies nativas de Polylepis estudiadas es $\sim 30 \mathrm{~km}$. $P$. lanuginosa forma parte de los bosques altimontanos del norte de los Andes (Beltrán et al. 2009). Presenta una distribución local restringida en el área ecotonal entre bosque montano y pajonal del páramo, en los sectores norte y occidental del Macizo del Cajas, desde $2800 \mathrm{~m}$ hasta $3400 \mathrm{~m}$ s. n. m. P. reticulata es la especie con un área de distribución local más extensa dentro del Macizo del Cajas, pero es discontinua, con miles de rodales dispersos en el páramo, concentrados en un rango altitudinal en general entre 3700 y 4000 m s. n. m. Predominan los rodales de 1-10 ha, a menudo en sitios protegidos en laderas con alta pendiente y relieve accidentado (Coblentz and Keating 2008), incluso sobre depósitos rocosos a veces muy voluminosos. También se los encuentra en las márgenes de lagunas y riachuelos y, en las cotas de menor altitud, de arroyos y ríos. La distribución de $P$. weberbaueri es muy local, con rodales pequeños o medios, a veces degradados, situados a menor altitud que $P$. reticulata en los sectores norte y sur del Macizo del Cajas. $P$. incana ocupa preferentemente determinados valles en $\mathrm{U}$, donde constituye bosques asociados a las riberas y laderas vecinas de las cuencas altas de los sectores septentrional y occidental del Macizo del Cajas, por encima de $3500 \mathrm{~m} \mathrm{~s}$. n. m. Tiene presencia en áreas con suelos profundos, incluso encharcados, o con afloramientos rocosos. Se rarifica con la altitud y forma rodales aislados en algunos sectores del páramo próximos a $3900 \mathrm{~m} \mathrm{s.} \mathrm{n}$. $\mathrm{m}$. y conectados con los valles mencionados, como el del río Migüir. P. racemosa, es una especie exótica que se usa en localidades de alta montaña como cercos vivos y barreras rompevientos.

\section{Características foliares y del fuste}

Se tomaron muestras de 10-20 hojas maduras (expandidas totalmente) de ramas expuestas al sol de 6 a 10 individuos seleccionados al azar de poblaciones del área central del rango altitudinal de distribución de las cuatro especies de Polylepis nativas (rango altitudinal: P. reticulata: 3800-4000; P. incana: 3600-3800; P. weberbaueri: 3300-3400, y P. lanuginosa: 3000-3200 m s. n. m.), y de $P$. racemosa, de una localidad a $3150 \mathrm{~m}$ s. n. m. Las muestras se conservaron en frío en bolsas herméticas de plástico. Se procesaron mediante separación de los foliolos de cada hoja, que fueron 
fotografiados para medir con exactitud el área foliar total mediante un software de análisis de imágenes. Después, se las secó en estufa a $70{ }^{\circ} \mathrm{C}(48 \mathrm{~h})$ y se determinó la masa foliar individual de cada hoja. El área foliar específica (AFE) se determinó como el cociente entre el área y la masa foliar.

De cinco a 19 individuos seleccionados al azar de $P$. reticulata, $P$. incana y $P$. racemosa se extrajeron tacos de madera de longitud variable y $5.15 \mathrm{~mm}$ de diámetro. Para ello se usó una barrena Pressler, en puntos situados a una $1.30 \mathrm{~m}$ de altura del fuste. A cada taco se le midió el volumen, el peso fresco y el seco. La densidad de la madera se determinó como el peso seco por unidad de volumen fresco. Su contenido de agua (CA), como la proporción sobre el peso fresco.

\section{Composición florística y atributos ecosistémicos}

En 14 parcelas de superficie individual de $1000 \mathrm{~m}^{2}$, establecidas en bosques maduros y con buen estado de conservación de Polylepis representativos del área central de su distribución, se realizó un inventario exhaustivo del número de árboles (altura mayor a $3 \mathrm{~m}$ ), por especie, con un diámetro normal (DAP) mayor a $2.5 \mathrm{~cm}$. Se registró su DAP y su altura $(\mathrm{H})$. También se determinó la presencia de otras especies de plantas vasculares. La altura dominante $\left(\mathrm{H}_{\mathrm{d}}\right)$ se estimó como el valor medio de los 10 árboles más altos con fuste recto o con inclinación $\leq 20^{\circ}$. La biomasa aérea arbórea (BA; $\mathrm{Mg} \mathrm{C}$ / ha) se estimó mediante el método de Chave et al. (2005), para lo que se consideraron los valores de DAP, $\mathrm{H}$ y densidad de la madera de Polylepis obtenida en el presente estudio, y un contenido de carbono de la biomasa de 47.4 $\mathrm{kg} \mathrm{C} / \mathrm{kg}$ (Martin and Thomas 2011). Para otras especies arbóreas, la densidad de la madera procede de una base de datos mundial (Zanne et al. 2009). La diversidad ecosistémica de especies arbóreas se estimó mediante el índice de Shannon desde los valores de biomasa y densidad arbórea poblacional (D) de cada especie.

En 2007, se estableció una parcela permanente de $2500 \mathrm{~m}^{2}$ en un rodal de $P$. reticulata situado a 3960 m s. n. m. (25 subparcelas de $100 \mathrm{~m}^{2}$ ). Se realizó un inventario exhaustivo de los árboles de todas las especies arbóreas $(\mathrm{H}>3 \mathrm{~m})$ con $\mathrm{DAP}>2.5 \mathrm{~cm}$. Los árboles fueron marcados y se les midió su DAP y H. En 2014 se midió de nuevo el DAP y se clasificaron de forma visual los árboles en tres clases según su dominancia definida por la posición y el desarrollo de su copa respecto a sus vecinos inmediatos: 1) dominante, ocupa el dosel principal superior, con copa bien desarrollada y sin limitaciones en la luz que recibe; 2) codominante, ocupa también el dosel principal, pero con copa de tamaño menor que los árboles vecinos, menos desarrollada o limitada por éstos, y recibe luz lateral parcial, y 3) dominado, con copa situada bajo el dosel principal y a una altura inferior a los árboles codominantes, poco desarrollada y a veces paupérrima, que no recibe luz cenital directa. También se registraron los árboles muertos.

Se determinó la biomasa aérea de Polylepis en ambas fechas mediante el método de Chave et al. (2005). En 2014, se estimó H mediante un modelo alométrico generado desde las medidas de H y DAP de los árboles de 2007. La tasa de secuestro de carbono promedio anual del periodo $\left(\mathrm{Mg} \mathrm{C} \cdot \mathrm{ha}^{-1} \cdot \mathrm{año}^{-1}\right)$ en este compartimento del ecosistema se estimó como el valor medio de las subparcelas.

\section{Análisis estadísticos}

Las diferencias en las características morfofuncionales de las especies se analizaron con la prueba U de Mann-Whitney mediante comparaciones por pares. Para estimar la relación entre variables morfo-funcionales y atributos ecosistémicos se llevaron a cabo análisis de regresión no lineal.

\section{Resultados}

\section{Diferencias morfo-funcionales foliares}

Las diferencias morfo-funcionales foliares de las especies presentes en el Macizo del Cajas se muestran en la Figura 1. Existen cuatro grupos por sus diferencias de área foliar (Figura 1a). $P$. reticulata y $P$. incana presentan los menores valores de área foliar, inferior a $5 \mathrm{~cm}^{2}, \mathrm{y} P$. lanuginosa el valor más alto, $17.3 \mathrm{~cm}^{2}$ (3.8 a 5.8 veces superior). Por su parte, $P$. weberbaueri muestra un valor intermedio, más próximo a las dos primeras especies y alrededor de $40 \%$ del valor de $P$. lanuginosa. $P$. racemosa difiere de los tres grupos previos y presenta un valor equivalente a $65 \%$ de $P$. lanuginosa.

También se diferencian cuatro grupos según su masa foliar (Figura 1b), aunque no idénticos a los previos. P. incana presenta una masa foliar de $\sim 40 \mathrm{mg}$, aproximadamente $50 \%$ de la masa foliar de $P$. reticulata y $P$. weberbaueri, que no difieren entre sí. P. lanuginosa muestra 
la masa foliar más alta; supera los $200 \mathrm{mg}$ y es significativamente mayor que las dos especies del grupo 'reticulata' (2.6 a 2.8 veces superior). $P$. racemosa muestra un valor intermedio y es superior al valor de las especies del grupo 'reticulata'.

El área foliar específica, que integra al área y a la masa foliar, define dos grandes grupos morfo-funcionales. El primero es monoespecífico (integrado por $P$. reticulata),

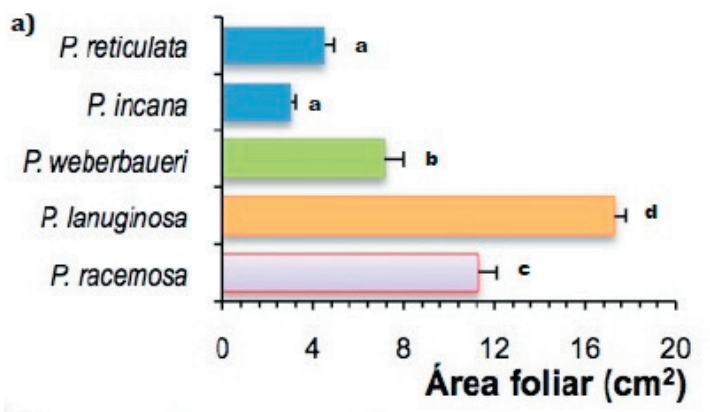

b)

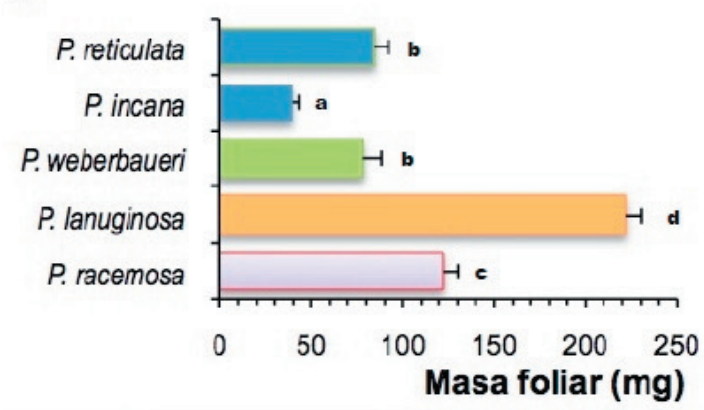

c)

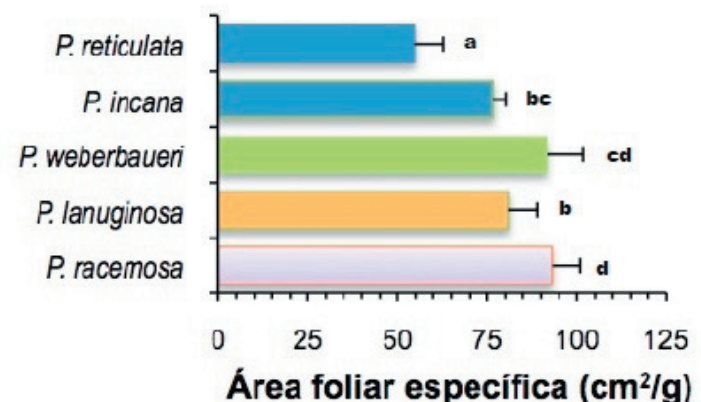

Figura 1. Valores medios ( \pm error estándar) de: (a) área foliar (AF), (b) masa foliar (MF) y (c) área foliar específica (AFE) de especies nativas del género Polylepis del Macizo del Cajas. Como referencia se indican los valores de una especie introducida ( $P$. racemosa). Letras distintas indican diferencias significativas (AF: $P<0.05$, MF y AFE: $P<0.01)$.

Figure 1. Mean values ( \pm standard error) of (a) leaf area (AF), (b) leaf mass (MF), and (c) specific leaf area (AFE) of native species of the genus Polylepis in the Cajas Massif. Values of an introduced species ( $P$. racemosa) are indicated as a reference. Different letters indicate significant differences (AF: $P<0.05, \mathrm{MF}$ and AFE: $P<0.01$ ). con un área foliar específica baja $\left(54.6 \mathrm{~cm}^{2} /\right.$ g). El segundo está compuesto por el resto de las especies, con un área foliar específica significativamente mayor (40-70\% más alto) que el de $P$. reticulata (Figura 1c).

\section{Contenido de agua y densidad del fuste}

La Tabla 1 muestra las diferencias de contenido de agua del fuste y densidad de la madera de dos especies nativas de Polylepis y de una introducida. El fuste de Polylepis muestra un alto contenido de agua, siempre superior a $55 \%$. $P$. reticulata y $P$. incana no difieren en esta característica, que es un $5 \%$ menor que la de $P$. racemosa. Polylepis presenta una densidad de la madera media-baja o ligera. La densidad de $P$. reticulata es la más alta (10\% más que la de $P$. incana y $17 \%$ más que la de $P$. racemosa). La Figura 2 muestra la relación interespecífica no lineal inversa entre el contenido de agua y la densidad de la madera observada en los árboles. Un incremento del $15 \%$ en la densidad determina una reducción promedio de $6 \%$ en el contenido de agua.

\section{Composición y diversidad florística de bosques}

Los bosques de Polylepis del Macizo del Cajas presentan una composición florística singular y muy variable. No son monoespecíficos. En el conjunto de bosques de $P$. reticulata y $P$. incana se han registrado 26 especies arbóreas que varían en su composición. La heterogeneidad entre rodales de $P$. reticulata es algo mayor que en los de $P$. incana. Estos bosques presentan una alta densidad total de árboles. Las poblaciones de Polylepis presentan al menos 400 individuos/ha, aunque suelen poseer más de 1000 individuos/ha (Tabla 2). Además, se

Tabla 1. Densidad de la madera y contenido de agua de dos especies nativas del género Polylepis y de una especie introducida, $P$. racemosa, en el Macizo del Cajas. Valores medios ( \pm error estándar). *: valores significativamente más altos

Table 1. Mean values ( \pm standard error) of stem wood density and stem water content of two native species of the genus Polylepis and one introduced species, P. racemosa, in the Cajas Massif. *: significantly higher values.

\begin{tabular}{lccc}
\hline Especie & $\mathrm{N}$ & $\begin{array}{c}\text { Densidad } \\
\left(\mathrm{kg} / \mathrm{m}^{3}\right)\end{array}$ & $\begin{array}{c}\text { Contenido de } \\
\text { agua }(\%)\end{array}$ \\
\hline P. reticulata & 17 & $503 \pm 10 \mathrm{a}^{*}$ & $55.1 \pm 0.7 \mathrm{a}$ \\
P. incana & 19 & $469 \pm 8 \mathrm{~b}$ & $55.8 \pm 0.5 \mathrm{a}$ \\
P. racemosa & 5 & $428 \pm 7 \mathrm{c}$ & $58.3 \pm 0.9 \mathrm{~b}^{*}$ \\
\hline
\end{tabular}

Letras diferentes indican diferencias significativas entre especies identificadas mediante la prueba $U$ de Mann-Whitney (contenido de agua: $P<0.05$; densidad: $P<0.01)$. 
han registrado entre 2 y 15 especies arbóreas por parcela, algunas con una densidad notable (200-400 individuos/ha), a veces más abundantes que Polylepis. La densidad de otras especies arbóreas puede constituir más del $80 \%$ del total. La diversidad arbórea de bosques de

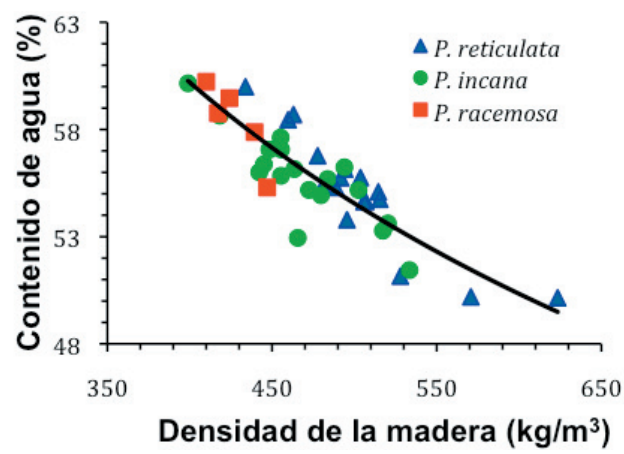

Figura 2. Relación entre el contenido de agua (CA) y la densidad de la madera (d) del fuste en árboles de diferentes especies de Polylepis. La línea de regresión representa la ecuación $\mathrm{CA}=847 \mathrm{~d}^{-0.441}\left(\mathrm{~N}=41, \mathrm{r}^{2}=0.77\right.$, $P<0.001)$.

Figure 2. Relationship between stem water content (CA) and stem wood density (d) in trees of different species of Polylepis. Regression line represents the equation $\mathrm{CA}=847 \mathrm{~d}^{-0.441}\left(\mathrm{~N}=41, \mathrm{r}^{2}=0.77, P<0.001\right)$.
$P$. reticulata y $P$. incana siempre es mayor si se estima desde el valor de densidad de árboles (hasta 3.08 bits) (Figura 3) que desde la biomasa aérea poblacional debido a la dominancia en la biomasa aérea arbórea total de Polylepis (en la mayoría de los casos, mayor al 70-80\%); se suelen alcanzar valores de diversidad arbórea entre 0.20 y 0.90 bits. En cambio, en el bosque estudiado de $P$. lanuginosa, la proporción de individuos y biomasa aérea arbórea de esta especie representa el 20-30\% del total (Tabla 2). En este bosque, la diversidad arbórea estimada es mayor que en los de $P$. reticulata y $P$. incana: 3.48 bits desde valores de D y 3.04 bits desde BA poblacional.

La diversidad de especies arbóreas de bosques de Polylepis muestra un patrón de disminución no lineal al aumentar la densidad de Polylepis (Figura 4). La riqueza de plantas de estos ecosistemas forestales es notable. Cuentan con más de 60 especies de angiospermas (31 familias, predominando Asteraceae y Rosaceae), y al menos 19 especies de pteridófitas (siete familias, predominando Drypteridaceae y Polypodiaceae).

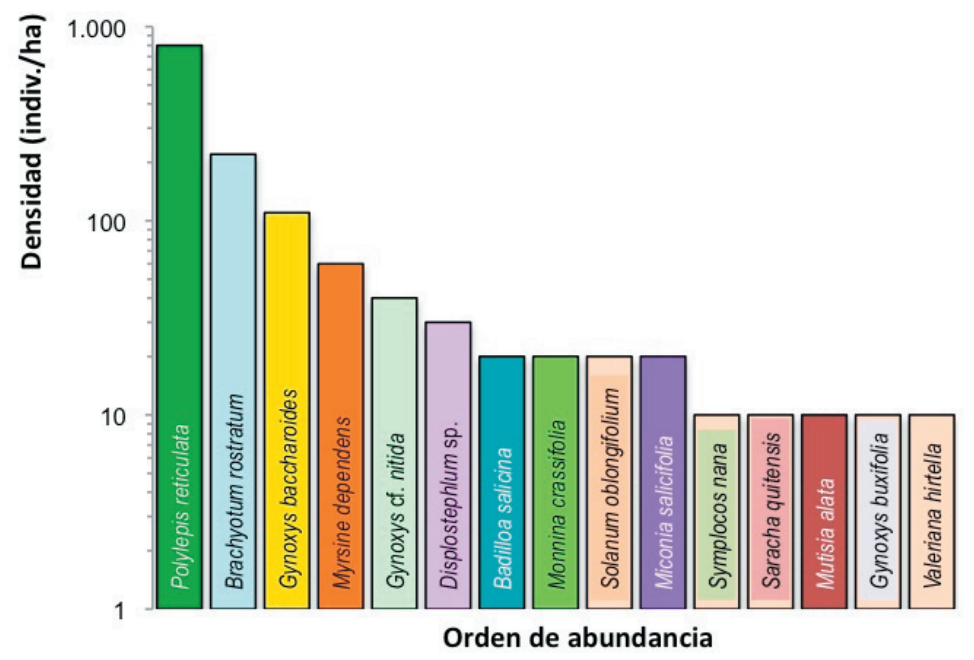

Figura 3. Composición de especies arbóreas de un bosque Polylepis reticulata ubicado a $3585 \mathrm{~m}$ s. $\mathrm{n}$. $\mathrm{m}$. en la cuenca alta del río Machángara, al este del Macizo de las Cajas. La diversidad de especies arbóreas es 2.24 bits (parcela de $1000 \mathrm{~m}^{2}$ ). P. reticulata representa el $58 \%$ de la densidad de árboles y el $97 \%$ de la biomasa aérea.

Figure 3. Tree species composition of a Polylepis reticulata woodland located at $3585 \mathrm{~m}$ a. s. 1 . in the upper basin of the Machángara River, east of the Cajas Massif. Tree species diversity is 2.24 bits $\left(1000 \mathrm{~m}^{2}\right.$ plot $)$. P. reticulata accounts for $58 \%$ of tree density and $97 \%$ of aboveground biomass.

Tabla 2. Rangos de variación de la estructura física y biomasa aérea de bosques de Polylepis (N) en el Macizo del Cajas situados entre 3500 y $3900 \mathrm{~m} \mathrm{s.} \mathrm{n.} \mathrm{m.,} \mathrm{excepto} \mathrm{el} \mathrm{de} \mathrm{P.} \mathrm{lanuginosa,} \mathrm{situado} \mathrm{a} 3300 \mathrm{~m} \mathrm{s.} \mathrm{n.} \mathrm{m.} \mathrm{Se} \mathrm{indican} \mathrm{los} \mathrm{valores} \mathrm{de}$ densidad de árboles $(\mathrm{D})$, diámetro normal (DAP), altura dominante $\left(\mathrm{H}_{\mathrm{d}}\right)$ y biomasa aérea $(\mathrm{BA})$ de Polylepis y total, y su proporción con respecto a la densidad total de árboles y la BA registrada en parcelas de $1000 \mathrm{~m}^{2}$.

Table 2. Variation ranges of the physical structure and aboveground biomass of Polylepis woodlands (N) in the Cajas Massif between 3500 and $3900 \mathrm{~m}$ a. s. 1., except for P. lanuginosa, located at $3300 \mathrm{~m}$ a. s. 1. The values of tree density (D), diameter at breast height (DAP), dominant height $\left(\mathrm{H}_{\mathrm{f}}\right)$ and total and Polylepis aboveground biomass (BA), and its proportion relative to the total tree density and BA recorded in plots of $1000 \mathrm{~m}^{2}$ are indicated.

\begin{tabular}{|c|c|c|c|c|c|c|c|c|c|}
\hline & $\mathrm{N}$ & $\begin{array}{c}\text { D } \\
\text { (103ind./ } \\
\text { ha) }\end{array}$ & $\begin{array}{c}\text { D relativa } \\
\text { (\% dens. } \\
\text { total) }\end{array}$ & $\begin{array}{l}\text { DAP } \\
\text { medio } \\
(\mathrm{cm})\end{array}$ & $\begin{array}{c}\text { Clase } \\
\text { diamétrica } \\
\text { modal }(\mathrm{cm})\end{array}$ & $\begin{array}{l}\mathrm{H}_{\mathrm{d}} \\
(\mathrm{m})\end{array}$ & $\begin{array}{c}\text { BAde } \\
\text { Polylepis } \\
\text { (MgC/ha) }\end{array}$ & $\begin{array}{l}\text { BA total } \\
\text { (Mg C/ha) }\end{array}$ & $\begin{array}{c}\text { BAde } \\
\text { Polylepis } \\
\text { (\% BA total) }\end{array}$ \\
\hline P. reticulata & 8 & $0.4-4.5$ & $15-95$ & $9.3-27.7$ & $5-10$ y $15-20$ & $7.0-14.3$ & $14-70$ & $38-97$ & $36-97$ \\
\hline P. incana & 5 & $0.7-2.7$ & $62-100$ & $15.9-35.6$ & $10-15$ y $25-30$ & $6.6-13.4$ & $31-209$ & $31-219$ & $96-100$ \\
\hline P. lanuginosa & 1 & 0.7 & 22 & 15.6 & 5-10 & 11.2 & 23 & 75 & 30 \\
\hline
\end{tabular}




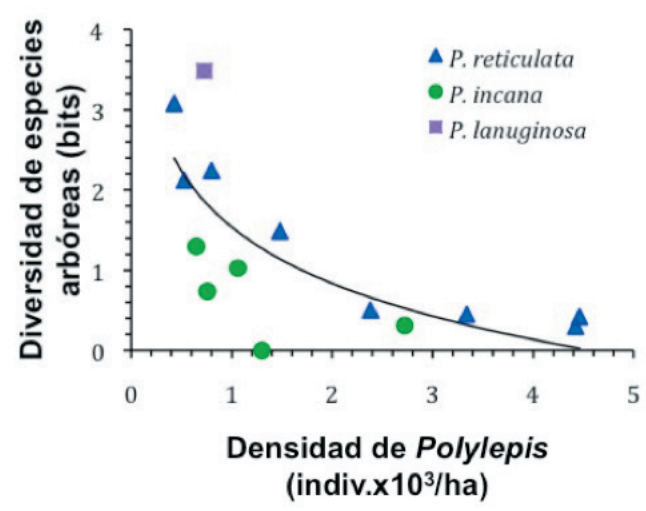

Figura 4. Patrón de disminución de la diversidad de especies arbóreas $\left(\mathrm{H}^{\prime}\right)$ de bosques de Polylepis en el Macizo del Cajas con el aumento de la densidad de árboles (D) de especies de este género. Ver Tabla 2. La curva de regresión representa la ecuación $\mathrm{H}^{\prime}=-1.013 \ln (\mathrm{D})+1.54$ $\left(\mathrm{N}=14 ; \mathrm{r}^{2}=0.54 ; \mathrm{P}<0.01\right)$.

Figure 4. Pattern of decreasing tree species diversity $\left(\mathrm{H}^{\prime}\right)$ of Polylepis woodlands in the Cajas Massif with the increase of tree density (D) of species of this genus. See Table 2. The regression curve represents the equation $\mathrm{H}^{\prime}=-1.013 \ln (\mathrm{D})+1.54\left(\mathrm{~N}=14, \mathrm{r}^{2}=0.54, P<0.01\right)$.

\section{Estructura física y biomasa de bosques}

La Tabla 2 muestra la variabilidad de la estructura física y biomasa aérea arbórea de bosques de Polylepis. Existe una alta heterogeneidad estructural entre rodales, derivada de una elevada variabilidad en densidad, estructura de clases diamétricas y tamaño medio de los árboles. La altura dominante varía entre 7 y $14 \mathrm{~m}$. Los árboles de $P$. lanuginosa del bosque estudiado que predominan son delgados, con un DAP entre 5 y $10 \mathrm{~cm}$. Esta clase diamétrica también predomina en bosques de $P$. reticulata, aunque a veces, la mayor proporción de árboles es de un tamaño mayor (entre 15 y $20 \mathrm{~cm}$ ). Los bosques de $P$. incana presentan árboles con un diámetro medio siempre mayor a $15 \mathrm{~cm}$ y una proporción mayor de árboles más gruesos que los bosques de $P$. reticulata y $P$. lanuginosa.

En general, la biomasa aérea de Polylepis es relativamente importante, aunque muy variable. En un bosque de $P$. reticulata, esta especie presenta una densidad baja (430 individuos/ha), un diámetro medio de 27.7 $\mathrm{cm}$ y una biomasa aérea de $57 \mathrm{Mg} \mathrm{C/ha}$. Sin embargo, otro bosque de $P$. reticulata con una densidad 3.7 veces superior (1600 individuos/ ha), aunque su diámetro medio es inferior $(16.3 \mathrm{~cm}$, un $58 \%$ del diámetro medio del caso anterior), presenta una biomasa aérea arbórea algo superior: $63 \mathrm{Mg}$ C/ha. La biomasa aérea arbórea total es, en general, aun mayor y también muy variable. Se destaca el alto valor de biomasa aérea total de un bosque de P. incana: $219 \mathrm{Mg} \mathrm{C/ha.} \mathrm{En} \mathrm{términos} \mathrm{generales,}$ en los bosques de $P$. reticulata y $P$. incana, las otras especies arbóreas distintas de Polylepis, aunque representan hasta el $75 \%$ de los individuos, contribuyen menos a la biomasa aérea (hasta el 64\%, pero frecuentemente menos) debido a la menor densidad o al tamaño individual de los árboles de estas especies arbóreas. En cambio, en el bosque altimontano de $P$. lanuginosa, la mayoría de los árboles de esta especie es codominante o superada en altura en el dosel por otras como Oreopanax andreanus, Weinmannia fagaroides o Sessea corymbosa. Los bosques de P. reticulata y $P$. incana a menudo presentan árboles con un fuste inclinado más de $20^{\circ}$. En los bosques más maduros de $P$. incana aparecen árboles con un DAP $>20 \mathrm{~cm}$ tendidos sobre el suelo y fustes de otros árboles - vivos y con fuste retorcido y enmarañado con el de otros árboles, casi reptantes - que forman con el volumen de su copa remanente un dosel más bajo, con ramas rectas, y dejan claros entre los que se desarrollan otras especies arbóreas.

\section{Cambios estructurales y secuestro de carbono en un rodal de $\mathrm{P}$. reticulata}

El inventario exhaustivo de la parcela permanente determinó la presencia de 926 árboles de $P$. reticulata, equivalente a 3704 individuos/ha. $\mathrm{Su}$ edad es joven-intermedia: el $41 \%$ de los árboles posee un DAP entre $5 \mathrm{y}$ $10 \mathrm{~cm}$, y el $46 \%$, entre 10 y $20 \mathrm{~cm}$. Tras siete años, debido al crecimiento individual de los árboles, se modificó levemente la estructura de clases diamétricas; disminuyó la frecuencia relativa de árboles menores de $10 \mathrm{~cm}$ (del $46 \%$ al $43 \%$ ) y aumentó la de los árboles más gruesos, de 15 a $35 \mathrm{~cm}$ (del 21\% al 26\%). Además, se mantuvo la proporción de la clase intermedia de 10-15 cm en un 31\%. El 52\% de los árboles tiene una altura entre 8 y $10 \mathrm{~m}$, y el $21 \%$, entre 10 y $12 \mathrm{~m}$. El 37\% de los árboles es de la clase dominado, el $32 \%$ es codominante $\mathrm{y}$ el tercio restante es dominante.

El cambio estructural en el periodo 20072014 supuso una mortalidad de 45 árboles, equivalente a 180 individuos/ha, que permite estimar una tasa de mortalidad anual promedio de $6.9 \%$. Esta tasa varía según la clase de dominancia de los árboles: 78\% ocurre en los árboles dominados, $18 \%$ en los codominantes y sólo $4 \%$ en los dominantes.

Además de $P$. reticulata, en la parcela permanente se registraron 34 árboles de 
otras seis especies (de los géneros Gynoxys, Diplostephium, Miconia, Monnina y Valeriana). Con ellos, la densidad arbórea total se eleva a 3960 individuos/ha, ya que $P$. reticulata representa el 92.3\%. El 53\% de los árboles de otras especies pertenece a la clase dominado, mientras que el $38 \%$ es codominante. La mortalidad en este grupo de especies se concentra también en la clase dominado. La tasa de mortalidad anual promedio estimada para el conjunto de estas otras especies es $20.1 \%$, casi tres veces superior a la de $P$. reticulata.

El modelo alométrico generado entre $\mathrm{H}$ y DAP de $P$. reticulata $\left(\mathrm{H}=1.46 \mathrm{DAP}^{0.747} ; \mathrm{r}^{2}=0.44\right.$; $P<0.001)$ permitió estimar la biomasa aérea en 2014 y una tasa de secuestro de carbono (mediaterror estándar) promedio en el período

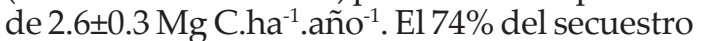
procede de árboles con un DAP inicial entre 10 y $20 \mathrm{~cm}$. Este almacenamiento de carbono resulta de una tasa media de crecimiento diametral, ponderada según la distribución de frecuencias de clases diamétricas, de $1.2 \mathrm{~mm} /$ año (equivalente a una tasa media ponderada de secuestro de $1.2 \mathrm{~kg}$ C/año por árbol).

\section{Discusión}

\section{Adaptación morfo-funcional de Polylepis}

Nuestros resultados revelan, por primera vez para los Andes tropicales húmedos de Ecuador, la misma tendencia de variación interespecífica a escala local en especies de Polylepis (Figura 1a). P. lanuginosa, una de las especies de Polylepis con mayor área foliar, ocupa hábitats de clima menos frío del Macizo del Cajas. Por el contrario, P. incana y $P$. reticulata, que presentan los valores de área foliar más bajos, ocupan hábitats de clima más frío; en particular, la segunda. $P$. weberbaueri presenta un área foliar coherente con su distribución altitudinal intermedia entre estas dos especies y $P$. lanuginosa. El área foliar de $P$. racemosa es coherente con su rango de distribución natural en Perú, que incluye localidades de altitud media (Mendoza and Asunción 2012). Los valores de área foliar de $P$. reticulata y $P$. incana del páramo del Macizo del Cajas son mayores que los de otras especies de este género de la puna seca de Bolivia y del norte de Chile (Macek et al. 2009). El área foliar de $P$. lanuginosa también supera al de $P$. lanata de sitios menos fríos de Bolivia (Hertel and Wesche 2008). La masa foliar, como indicador alternativo del tamaño foliar, es coincidente (Figura $1 b$ ), excepto que $P$. weberbaueri no se diferencia de $P$. reticulata, y $P$. incana tiene la menor masa observada, debida a foliolos más delgados que las especies anteriores. Nuestros resultados coinciden con reportes previos de una reducción del área foliar con la altitud, lo que sugiere que el área foliar de las especies de Polylepis es una adaptación ambiental con base filogenética. A modo de ejemplo, nueve especies de Polylepis procedentes de varias altitudes y áreas climáticas presentan, en condiciones controladas de cultivo, una tendencia interespecífica de reducción del área foliar al disminuir la temperatura media anual de las localidades de origen (puna húmeda y seca de Perú y Bolivia, y un área subtropical de la Argentina) (Toivonen et al. 2014). Poblaciones silvestres de tres especies de Polylepis de la puna seca y húmeda de Bolivia y Chile tienen menor área foliar que otras de menor altitud (Hertel and Wesche 2008; Maceck et al. 2009). El área foliar disminuye con la altitud en $P$. sericea del páramo de Venezuela (Colmenares-Arteaga et al. 2005).

El significado adaptativo de un bajo área foliar a mayor altitud en especies de Polylepis se interpreta como protección ante el estrés por frío, déficit hídrico o alta radiación solar (Maceck et al. 2009). Un área foliar bajo conlleva una menor superficie de intercepción de radiación e inferior asimilación de $\mathrm{CO}_{2}$, transpiración y crecimiento (ColmenaresArteaga et al. 2005). Precisamente, Azócar et al. (2007) demostraron cómo la resistencia a heladas en $P$. sericea, a diferencia de otras especies de climas más extremos como $P$. tarapacana y $P$. australis, resulta de un mecanismo de evasión de la congelación por super-enfríamiento. Este mecanismo ecofisológico protector podría ser compartido por $P$. reticulata y $P$. incana, propias de clima tropical húmedo y frío.

El área foliar específica, en cambio, no permite discriminar las especies (Figura 1c), como observan Toivonen et al. (2014) en condiciones experimentales. Sin embargo, la especie que alcanza mayor altitud, $P$. reticulata, presenta un área foliar específica significativamente más bajo. Las restantes especies se pueden agrupar por su área foliar específica relativamente alto, lo que sugiere una compleja variabilidad subyacente y efectos compensatorios en las adaptaciones foliares, probablemente con una limitación genética. El área foliar específica de $P$. reticulata es similar al de una población de $P$. sericea del páramo venezolano situada a $4100 \mathrm{~m} \mathrm{~s}$. n. m. (Colmenares-Arteaga et al. 2005). El área foliar 
específica del resto de las especies es semejante al que exhiben $P$. sericea, a menor altitud, y $P$. cuadrijuga, en el páramo colombiano (Ramos et al. 2013). Los valores de área foliar específica de las especies de Polylepis en el Macizo del Cajas son mayores que los de otras especies del género en áreas más secas de la puna (Hoch and Körner 2005; Macek et al. 2009).

Las hojas más gruesas y con menor superficie fotosintética por unidad de masa foliar presentan un coste energético de construcción y mantenimiento relativamente alto y menor tasa de crecimiento (Villar et al. 2013). Esto es coherente con el área foliar específica relativamente alto de $P$. racemosa y su mayor tasa de crecimiento en comparación con otras especies de Polylepis (Pretell et al. 1985). Es probable que la disminución del área foliar específica con la altitud (Körner 2012) también se relacione con la distribución local de las especies de Polylepis, ya que está inversamente correlacionada con el punto de compensación para la luz de la fotosíntesis y la xeromorfia foliar (Macek et al. 2009; Toivonen et al. 2014). Un área foliar específica alta sería una ventaja ecológica para ocupar hábitats menos fríos y, en particular, con mayor disponibilidad de recursos edáficos que las cotas más altas del páramo. Implicaría una mayor capacidad competitiva con otras especies arbóreas más probables a menor altitud.

El valor del área foliar específica permite inferir propiedades a nivel ecosistema en la mayoría de los bosques donde Polylepis es absolutamente dominante (Garnier et al. 2004; Poorter et al. 2009). Los valores de área foliar específica de $P$. reticulata y $P$. incana son inferiores a la mediana de las especies arbóreas perennifolias (entre 20\% y 40\%). El resto de especies de Polylepis son similares. Los valores medios de AFE de todas las especies de Polylepis del Macizo del Cajas son inferiores a la mediana de especies de otros tipos de bosques tropicales (entre 32\% y 60\%) (Poorter et al. 2009) y son indicadores de ecosistemas cuya producción primaria está limitada por agua y nutrientes, lo cual se asocia al frío y a la aridez climática entre 3000 y 4000 m s.n.m. En este rango altitudinal se concentran la mayoría de las especies de Polylepis en Bolivia y Perú (Mendoza and Cano 2012), y también en Ecuador (Romoleroux 1992, 1996).

Los resultados refuerzan la hipótesis de trabajo enunciada sin constituir una prueba experimental. No obstante, existe una complejidad adaptativa que requiere estudios más profundos debido a la amplitud de tolerancia ambiental de Polylepis (Mendoza and Cano 2012), a la plasticidad fenotípica de sus especies (Toivonen et al. 2014) y a la diversidad de los factores climáticos que controlan su distribución geográfica (e.g., Kessler et al. 2014; Cuyckens 2016). Sin embargo, la vulnerabilidad de las especies de Polylepis al cambio climático puede ser diferencial: son más sensibles las de cotas bajas, en especial $P$. lanuginosa, cuya capacidad de migración ascendente es probablemente inferior a la de otras especies del bosque montano por su menor tasa de crecimiento.

El alto contenido de agua del fuste de Polylepis (Tabla 1) se interpreta como una capacidad de almacenamiento análoga a la adaptación a aridez climática de algunas especies arbóreas del bosque tropical seco (Borchert 1994). Las especies de Polylepis del Macizo del Cajas presentan una densidad de la madera mediabaja o ligera, tal como han observado otros autores (Fehse et al. 2002). Es inferior a la densidad de madera media de las especies arbóreas de los bosques neotropicales (entre $22 \%$ y 34\%) (Chave et al. 2006). La relación interespecífica no lineal e inversa entre el contenido de agua y densidad de la madera (Figura 1) es coherente con la restricción en la capacidad de almacenamiento de agua al disminuir los espacios libres intracelulares y de las paredes celulares en los tejidos del fuste de los árboles con el aumento de la densidad de la madera (Simpson 1993). Además, es similar a la observada por Borchert (1994). Las diferencias en densidad del fuste, pero no en contenido de agua, entre $P$. reticulata y $P$. incana sugieren que otras características funcionales condicionan su distribución.

La capacidad de almacenamiento de agua podría ser una característica de las especies de Polylepis, al menos de aquéllas de hábitats de mayor altitud con aridez climática o estacionalidad hídrica. Sería una especialización ecológica que permite una mayor eficiencia en el uso de los recursos y un acoplamiento ecofisiológico con las condiciones rigurosas de alta altitud, convergente con las características xeromórficas foliares. Sin embargo, en Polylepis tarapacana se observaron valores de densidad de madera superiores a los registrados en especies del presente estudio, alrededor de $600 \mathrm{~kg} / \mathrm{m}^{3}$, y una tendencia a disminución al aumentar la altitud (Hoch and Körner 2005), quizá por ocupar hábitats extremos de la puna seca boliviana (entre 
4300 y 4800 m s. n. m.). En esta especie de la puna, la asociación entre la alta densidad de la madera, la baja área foliar específica y un crecimiento diametral medio limitado (Domic and Capriles 2009) es coherente con la observación de lo contrario en $P$. reticulata en el páramo ecuatoriano (Tabla 1 y Figura 1), y una tasa de crecimiento moderada.

\section{Estructura y dinámica de ecosistemas de Polylepis}

Las especies de Polylepis del Macizo del Cajas son pioneras y heliófilas, y se establecen y crecen mejor que otras especies en hábitats pobres en recursos y con una competencia nula o limitada por parte de otras especies de plantas vasculares (observación personal). Tras el establecimiento de una cohorte densa de plántulas de Polylepis en estos hábitats (e.g., deslizamiento deladeras o depósitos coluviales rocosos) se desarrollan bosques de alta densidad arbórea. P. lanuginosa, por su carácter más xerófilo que otras especies arbóreas del bosque altimontano, puede establecerse en hábitats expuestos a vientos desecantes, alta radiación solar y menor disponibilidad de agua. Se genera una población coetánea que se hace más irregular por el desarrollo de algunos árboles en micrositios más favorables. La alta densidad limita el crecimiento individual y la acumulación de BA por la competencia intraespecífica por los recursos (Pretzsch and Biber 2005). Los árboles dominados mueren por autoaclareo y disminuye la densidad poblacional, tal como se registró. Estas especies de Polylepis son longevas; persisten hasta la madurez del bosque y preservan su dominancia en el dosel.

La composición florística del bosque de páramo depende de la dinámica sucesional y de la variabilidad altitudinal y topográfica, y, al aumentar la altitud, cambia la composición y disminuye la diversidad vegetal (Kessler 2006). Expresa el efecto del incremento de la rigurosidad ambiental y de una reducción de la conectividad con el bosque montano (Figuras 3 y 4). Desde 3400 a 3600 m s. n. m. desaparecen especies como Myrsine andina, M. dependens, Vallea stipularis, Oreopanax aviceniifolius, Weinmannia fagaroides, Badilloa salicina, mientras que Sessea crassivenosa. Miconia salicifolia y Gynoxys cuicochensis se mantienen. A mayor altitud y aislamiento, en los bosques de Polylepis aparecen especies heliófilas de páramo arbustivo, a veces con talla arbórea (e.g., Diplostephium espinosae, D. glandulosum, Valeriana hirtella y Monnina crassifolia). Aquellos bosques con dosel más alto y árboles gruesos albergan, incluso, especies esciófilas como Gynoxys cf. latifolia. La composición florística del sotobosque es similar a la registrada por Romoleroux et al. (2008). Entre ellas, existen 12 especies vegetales endémicas y especies especialistas forestales que no se hallan en otros tipos de vegetación del páramo (Minga and Verdugo 2007).

La relación entre diversidad arbórea y densidad de Polylepis (Figura 4) sugiere una interdependencia entre la estructura física del ecosistema y la composición florística. Expresa sintéticamente que si la densidad de árboles de Polylepis en un bosque de páramo o montano es baja, con individuos gruesos o viejos, la diversidad de especies es alta (bosques maduros o más conectados con áreas de bosque montano). Sin embargo, si la densidad de árboles de Polylepis es alta, con predominio de individuos delgados o jóvenes, la diversidad de especies es mucho más baja (bosques juveniles y de hábitats más extremos o aislados en el páramo).

\section{Conservación de especies de Polylepis y sus ecosistemas}

La biomasa aérea y secuestro de carbono, para unas condiciones climáticas húmedas del páramo de Ecuador, sugiere dos observaciones relevantes para la conservación y la valoración de los servicios ecosistémicos de los bosques de Polylepis. Primero, es destacable la importancia de este tipo de ecosistema forestal como almacén de carbono en la biomasa aérea (Tabla 2). El rango de valores de biomasa aérea registrados en el Macizo del Cajas comprende los observados en bosques de $P$. incana de diferente edad del páramo del centro de Ecuador (Fehse et al. 2002) y de la puna húmeda peruana (Vásquez et al. 2014). El valor de $219 \mathrm{Mg} \mathrm{C/ha}$ del presente estudio es el récord publicado de biomasa aérea para un bosque de Polylepis. En condiciones favorables, la biomasa aérea de un bosque de Polylepis puede ser similar o mayor a la de otros bosques tropicales, como algunos tipos de bosque montano (Spracklen and Righelato 2016). Segundo, aunque Polylepis es un género de crecimiento lento, los resultados de nuestro estudio, realizado con medidas del conjunto más numeroso de árboles y el período más largo de observación que se conocen para Polylepis, indica un crecimiento diametral ponderado estimado coherente con el observado en otra especie de Polylepis en un ambiente más desfavorable (Hoch and Körner 
2005; Domic and Capriles 2009). Además, es notable la tasa de secuestro de carbono a alta altitud y concordante con las tasas elevadas observadas en bosques de $P$. incana en otros páramos de Ecuador (Fehse et al. 2002).

La mejor forma de conservar las especies de Polylepis y su biodiversidad es preservar los bosques de los que forman parte. En el Parque Nacional Cajas, aunque con impactos locales en algunas zonas, existe una conservación adecuada de los bosques de páramo dominados por $P$. reticulata, cuya fragmentación natural en parte es paleoclimática (e.g., Rodríguez and Behling 2012). Sin embargo, gran parte de los bosques de $P$. incana, fundamentalmente en áreas no protegidas de menor altitud, padecieron y padecen efectos de fragmentación y otras formas de degradación antropogénica de su estructura física, y empobrecimiento de la composición florística del sotobosque. Las principales son uso ganadero, extracción de leña, e incluso la sustracción por viveristas de plántulas de $P$. incana. Por su distribución restringida, su identidad relíctica y el carácter endémico de $P$. lanuginosa, los bosques remanentes de $P$. lanuginosa y $P$. weberbaueri merecen una especial identificación y protección a fin de conservar su biodiversidad. En conclusión, en áreas de alta montaña deforestadas o degradadas, la conservación y la restauración ecológica de bosques de Polylepis, capaces de retener carbono en biomasa y suelos, es una opción valiosa para preservar la biodiversidad altoandina amenazada, para mitigar el cambio climático (Fehse et al. 2002; Vásquez et al. 2014) y para proveer otros servicios ecosistémicos.

Agradecimientos. El Ministerio de Agricultura y Pesca, Alimentación y Medio Ambiente de España, la Universidad del Azuay y la Fundación Matrix, Investigación y Desarrollo Sostenible han cofinanciado esta investigación, realizada bajo el permiso número MAECGZ6-DPAC-2015-1528. Daniel Renison y dos revisores anónimos contribuyeron a la claridad del trabajo.

\section{REFERENCIAS}

Azócar, A., F. Rada, and C. García-Núñez. 2007. Functional characteristics of the arborescent genus Polylepis along a latitudinal gradient in the high Andes. Interciencia 32:663-668.

Beltrán, K., S. Salgado, F. Cuesta, S. León-Yánez, K. Romoleroux, E. Ortiz, A. Cárdenas, and Velástegui, A. 2009. Distribución Espacial, Sistemas Ecológicos y Caracterización Florística de los Páramos en el Ecuador. EcoCiencia, Proyecto Páramo Andino y Herbario QCA. Quito, Ecuador.

Borchert, R. 1994. Soil and Stem Water Storage Determine Phenology and Distribution of Tropical Dry Forest Trees. Ecology 75:1437-1449.

Chave, J., C. Andalo, S. Brown, M.A. Cairns, J.Q. Chambers, D. Eamus, H. Folster, F. Fromard, N. Higuchi, T. Kira, J. P. Lescure, B. W. Nelson, H. Ogawa, H. Puig, B. Riera, and T. Kamakura. 2005. Tree allometry and improved estimation of carbon stocks and balance in tropical forests. Oecologia 145:87-99.

Chave, J., H. C. Muller-Landau, T. R. Baker, T. Easdale, H. ter Steege, and O. Campbell. 2006. Regional and phylogenetic variation of wood density across 2456 neotropical tree species. Ecological Applications 16:2356-2367.

Cierjacks, A., J. E. Iglesias, K. Wesche, and I. Hensen. 2007. Impact of sowing, canopy cover and litter on seedling dynamics of two Polylepis species at upper treelines in central Ecuador. Journal of Tropical Ecology 23:309-318.

Coblentz, D., and P. L. Keating. 2008. Topographic controls on the distribution of tree islands in the high Andes of south-western Ecuador. J Biogeogr 35:2026-2038.

Colmenares-Arteaga, M., F. Rada, and R. Luque. 2005. Anatomía foliar de Polylepis sericea Wedd. (Rosaceae) a dos altitudes en los Altos andes venezolanos. Plántula 3:141-148.

Cuyckens, G. A. E., D. A. Christie, A. I. Domic, L. R. Malizia, and D. Renison. 2016. Climate change and the distribution and conservation of the world's highest elevation woodlands in the South American Altiplano. Global and Planetary Change 137:79-87.

Domic, A. I., and J. M. Capriles. 2009. Allometry and effects of extreme elevation on growth velocity of the Andean tree Polylepis tarapacana Philippi (Rosaceae). Plant Ecol 205:223-234.

Fehse, J., R. Hofstede, N. Aguirre, Ch. Paladines, A. Kooijman, and J. Sevink. 2002. High altitude tropical secondary forests: a competitive carbon sink? Forest Ecology and Management 163:9-25.

Fjeldså, J. 2002. Polylepis Forest - vestiges of vanishing ecosystem in the Andes. Ecotropica 8:111-125.

Garnier, E., J. Cortez, G. Billes, M. L. Navas, C. Roumet, M. Debussche, G. Laurent, A. Blanchard, D. Aubry, and A. Bellmann. 2004. Plant functional markers capture ecosystem properties during secondary succession. Ecology 85: 2630-2637.

Gosling, W. D., J. A. Hanselman, C. Knox, B. G. Valencia, and M. B. Bush. 2009. Long term drivers of change in Polylepis woodland distribution in the central Andes. Journal of Vegetation Science 20:1041-1052.

Hertel, D., and K. Wesche. 2008. Tropical moist Polylepis stands at the treeline in East Bolivia: the effect of elevation on stand microclimate, above- and below-ground structure, and regeneration. Trees 22:303-315.

Hijmans, R. J., S. E. Cameron, J. L. Parra, P. G. Jones, and A. Jarvis. 2005. Very high resolution interpolated climate 
surfaces for global land areas. Int J Climatol 25:1965-1978.

Hoch, G., and C. Körner. 2005. Growth, demography and carbon relations of Polylepis trees at the world's highest treeline. Functional Ecology 19:941-951.

Kessler, M. 2000. Observations on a human-induced fire event at a humid timberline in the Bolivian Andes. Ecotropica 6:89-93.

Kessler, M. 2002. The "Polylepis problem": where do we stand? Ecotropica 8:97-110.

Kessler, M. 2006. Bosques de Polylepis. Pp. 110-120 in R. Moraes, B. Øllgaard, L. P. Kvist, F. Borchsenius and H. Balslev. (eds.). Botánica Económica de los Andes Centrales. La Paz, Bolivia.

Kessler, M., and A. N. Schmidt-Lebuhn. 2006. Taxonomical and distributional notes on Polylepis (Rosaceae). Organisms Diversity and Evolution 6:67-69.

Kessler, M., J. M. Toivonen, S. P. Sylvester, J. Kluge, and D. Hertel. 2014. Elevational patterns of Polylepis tree height (Rosaceae) in the high Andes of Peru: role of human impact and climatic conditions. Frontiers in Plant Science 194: $1-12$.

Körner, C. 2012. Alpine Treelines: Functional Ecology of the Global High Elevation Tree Limits. Springer, Berlin, Germany.

Macek, P., J. Macková, and F. de Bello. 2009. Morphological and ecophysiological traits shaping altitudinal distribution of three Polylepis treeline species in the dry tropical Andes. Acta Oecologica 35:778-785.

Martin, A. R., and S. C. Thomas. 2011. A Reassessment of Carbon Content in Tropical Trees. PLoS ONE 6:e23533. doi: 10.1371/journal.pone.0023533.

Mendoza, W., and A. Cano. 2012. El género Polylepis en el Perú. Taxonomía, Morfología y Distribución. Editorial Académica Española, Saarbrücken, Germany.

Minga, D., and A. Verdugo. 2007. Riqueza florística y endemismo del Parque Nacional Cajas. Informe técnico no publicado. Herbario Azuay, Universidad del Azuay, Cuenca, Ecuador.

Minga, D., R. Ansaloni, A. Verdugo, and C. Ulloa Ulloa. 2016. Flora del Páramo del Cajas. Universidad del Azuay, Cuenca, Ecuador.

Poorter, H., U. Niinemets, L. Poorter, I. J. Wright, and R. Villar. 2009. Causes and consequences of variation in leaf mass per area (LMA): a meta-analysis. New Phytol 182:565-588.

Pretell, J., D. Ocaña, R. Jon, and E. Barahona. 1985. Apuntes sobre algunas especies forestales nativas de la sierra peruana. Proyecto FAO/Holanda/Infor, Lima, Perú.

Pretzsch, H., and P. Biber. 2005. A re-evaluation of Reineke's rule and stand density index. Forest Science 51:304-320. Purcell, J., A. Brelsford, and M. Kessler. 2004. The World's Highest Forest. American Scientist 92:454-461.

Ramos, C., S. P. Buitrago, K. L. Pulido, and L. J. Vanegas. 2013. Variabilidad ambiental y respuestas fisiológicas de Polylepis cuadrijuga (Rosaceae) en un ambiente fragmentado en el Páramo de la Rusia (Colombia). Rev Biol Trop 61: 351-361.

Renison, D., I. Hensen, and R. Suárez. 2011. Landscape structural complexity of high-mountain Polylepis australis forests: A new aspect of restoration goals. Restoration Ecology 19:390-398.

Renison, D., G. A. E. Cuyckens, S. Pacheco, G. F. Guzmán, H. R. Grau, P. Márcora, G. Robledo, A. M. Cingolani, J. Dominguez, M. Landi, L. Bellis, and I. Hensen. 2013. Distribución y estado de conservación de las poblaciones de árboles y arbustos del género Polylepis (Rosaceae) en las montañas de Argentina. Ecología Austral 23:27-36.

Rodríguez, F., and H. Behling. 2012. Late Quaternary vegetation, climate and fire dynamics, and evidence of early to mid-Holocene Polylepis forests in the Jimbura region of the southernmost Ecuadorian Andes. Palaeogeography, Palaeoclimatology, Palaeoecology 350-352:247-25.

Romoleroux, K. 1992. Rosaceae in the Páramo of Ecuador. Pp. 85-94 in H. Balsey and J.L. Lutevb (eds.). Páramo: An Andean Ecosystem under Human Influence. Academic Press, Londres, United Kingdom.

Romoleroux, K. 1996. Rosaceae. Pp. 1-152 in G. Harling and L. Anderson (eds.). Flora of Ecuador. No. 56. University of Götenborg, Riksmuseum, Pontificia Universidad Católica del Ecuador; Göteborg, Stockholm, Quito.

Romoleroux, K., D. Cárate, R. Erler, and H. Navarrete. 2008. Los Bosques olvidados de los Andes. Nuestra Ciencia 10:34-37.

Schmidt'Lebuhn, A. N., J. Fuchs, D. Hertel, H. Hirsch, J. Toivonen, and M. Kessler. 2010. An Andean radiation: polyploidy in the tree genus Polylepis (Rosaceae, Sanguisorbeae). Plant Biology 12:917-926.

Segovia-Salcedo, M. C. 2011. Los riesgos de la reforestación de los páramos con especies exóticas: el caso de Polylepis racemosa. Propuestas andinas No. 4. CONDESAN, Quito, Ecuador.

Segovia-Salcedo, M. C., and P. Quijia-Lamiña 2013. Citogeografía de cuatro especies de Polylepis (Rosaceae) en el Ecuador: Información relevante para el manejo y conservación de los bosques andinos. Pp. 467-485 in F. Cuesta, J. Sevink, L. D. Llambí, B. de Bièvre and J. Posner (eds.). Avances en investigación para la conservación de los páramos andinos. CONDESAN, Quito, Ecuador.

Simpson, B. B. 1979. A revision of the genus Polylepis (Rosaceae: Sanguisorbeae). Smithson. Contrib. Bot. 43. Smithsonian Institution, Washington, D.C., USA.

Simpson, W. T. 1993. Specific gravity, moisture content, and density relationship for wood. Gen. Tech. Rep. FPL-GTR76. U.S. Department of Agriculture, Forest Service, Forest Products Laboratory, Madison, Wisconsin, USA.

Spracklen, D. R., and V. Righelato. 2016. Carbon storage and sequestration of re-growing montane forests in southern Ecuador. Forest Ecology and Management 364:139-144. 
Tejedor Garavito, N., A. C. Newton, and S. Oldfield. 2015. Regional Red List assessment of tree species in upper montane forests of the Tropical Andes. Oryx 49:397-409.

Tejedor Garavito, N., N. Álvarez, S. Arango Caro, A. Araujo Murakami, C. Blundo, T. E. Boza Espinoza, et al. 2012. Evaluación del estado de conservación de los bosques montanos en los Andes tropicales. Ecosistemas 21:148-166.

Toivonen, J. M., V. Horna, M. Kessler, K. Ruokolainen, and D. Hertel. 2014. Interspecific variation in functional traits in relation to species climatic niche optima in Andean Polylepis (Rosaceae) tree species: evidence for climatic adaptations. Functional Plant Biology 41:301-312.

Vásquez, E., B. Ladd, and N. Borchard. 2014. Carbon storage in a high-altitude Polylepis woodland in the Peruvian Andes. Alp. Botany 124:71-75.

Villar, R., J. Ruiz-Robleto, J. L. Ubera, and H. Poorter. 2013. Exploring variation in leaf mass per area (LMA) from leaf to cell: an anatomical analysis of 26 woody species. American Journal of Botany 100:1969-1980.

Zanne, A. E., G. López-González, D. A. Coomes, J. Ilic, S. Jansen, S. L. Lewis, R. B. Miller, N. G. Swenson, M. C. Wiemann, and J. Chave. 2009. Data from: Towards a worldwide wood economics spectrum. Dryad Digital Repository. http: //dx.doi.org/10.5061/dryad.234. 